\title{
Refining imaging tools to detect advanced fibrosis: could liver surface nodularity address an unmet need in the NAFLD epidemic?
}

\author{
Jörn M. Schattenberg ${ }^{1,2} \cdot$ Tilman Emrich $^{3,4,5}$
}

Received: 29 September 2021 / Revised: 5 November 2021 / Accepted: 8 November 2021 / Published online: 27 January 2022

(c) The Author(s) 2022

Dioguardi-Burgio et al [1] present a study that explores liver surface nodularity (LSN) on MRI to identify patients with advanced fibrosis related to non-alcoholic fatty liver disease (NAFLD). NAFLD is a highly prevalent liver disease with a high economic burden [2]. Still, the diagnosis rates are relatively low and even cirrhotic disease stages are only infrequently diagnosed. Today, there are multiple barriers that preclude the rapid identification of patients with advanced liver disease, even when they are under regular medical care, e.g., related to type 2 diabetes. Importantly, the mortality of patients living with NAFLD has been linked to the disease stages and excess mortality occurs in patients with advanced liver fibrosis stages defined as F3 and higher according to the NASH CRN staging classification [3].

In this retrospective cohort study at a tertiary care center in France, patients who underwent liver biopsy and received MRI as part of their clinical workup within 3 months were

Jörn M. Schattenberg and Tilman Emrich contributed equally to this work.

This comment refers to the article available at https://doi.org/10. 1007/s00330-021-08261-6.

Jörn M. Schattenberg

joern.schattenberg@unimedizin-mainz.de

1 Department of Internal Medicine I, University Medical Center of the Johannes Gutenberg-University, Langenbeckstrasse 1, 55131 Mainz, Germany

2 Metabolic Liver Research Program, University Medical Center of the Johannes Gutenberg-University, Mainz, Germany

3 Division of Cardiovascular Imaging, Department of Radiology and Radiological Science, Medical University of South Carolina, Charleston, SC, USA

4 Department of Diagnostic and Interventional Radiology, University Medical Center of the Johannes Gutenberg-University, Mainz, Germany

5 German Center for Cardiovascular Research (DZHK), Partner-Site Rhine-Main, Mainz, Germany included. Data on 142 patients were available and 37\% of these had advanced fibrosis defined as the histological disease stages F3 and F4. The AUC of LSN in this analysis was 0.838 [95CI 0.767-0.894]. These numbers are high, but there are important caveats that have to be considered when assessing diagnostic markers of liver fibrosis. The AUC is highly dependent on the quality of the reference standard-in this analysis liver biopsy-and confounders include the degree of fragmentation and the length of the biopsy [4]. Coming from expert centers, the highest quality can be assumed; however, aspects of the quality of biopsies are only infrequently reported [5]. The referral character of the involved centers impacts the pretest probability of the target condition and both the AUC [6] and the positive and negative predictive values will be different outside of this study population. Importantly, most non-invasive biomarkers for the assessment of liver fibrosis do not exceed 0.85 when compared to liver histology [7].

From a more general perspective, the use of MRI over CT appears favorable related to safety aspects, while in clinical care, many more patients do receive CT scans for a wide range of indications. The concept to use LSN to detect advanced liver fibrosis on CT scan has previously successfully shown for hepatic fibrosis of different indications [8], chronic viral hepatitis c [9], and portal hypertension [10]. Independent of the imaging modalities, it will be of great importance to report and acknowledge incidental hepatic steatosis on imaging. In the aftermath of the current analysis, it has to be considered to include LSN in radiology reports to improve the identification of asymptomatic at-risk patients with advanced liver disease. Therefore, training of both radiologists and physicians ordering radiological testing on the importance of NAFLD and more specifically the concept of advanced fibrosis as well as associated complications will be of great importance.

Currently, there are several non-invasive tests (NITs) being developed as diagnostic biomarkers to detect advanced fibrosis related to NAFLD [10]. Most current concepts aim 
at ruling out patients with a test that is widely available and comes at a low cost in an initial step, e.g., the FIB-4 score. Next, a more expensive test in an enriched population can be applied. This concept was also presented in the current study. By using LSN sequentially to FIB-4, 73\% could be ruled out as advanced fibrosis, whereas a comparable number of cases were ruled in in the developmental cohort. While these numbers are acceptable, the use of imaging biomarker over bloodbased biomarker carries the disadvantage of higher costs.

Beyond the use as a diagnostic biomarker, exploration of LSN as a prognostic biomarker seems appealing. In the realm of blood-based tests, recently the enhanced liver fibrosis (ELF) test received FDA approval. Looking at imaging modalities, magnetic resonance elastography (MRE) predicted the risk of future decompensation or death in cirrhosis with a 1-year probability of $9 \%$ with a baseline LSM above $5 \mathrm{kPa}$ and of $20 \%$ with MRE above $8 \mathrm{kPa}$ [11]. However, MRE is a technically challenging examination that requires dedicated equipment, training, and sequences. In contrast, LSN could be applied to any type of cross-sectional liver imaging.

The current study is remarkable for the following reasons. There is an urgent need to refine NITs and advance the field of diagnostic biomarkers [12]. As abdominal imaging (CT or MRI) is frequently performed, this could be one link to improve the diagnosis rates for a disease that is highly preventable but largely underdiagnosed. This will be of particular interest as the linkage to care will be further simplified with pharmacological options that are expected in 2023. On the other hand, the lack of awareness of NAFLD and hepatic fibrosis in most areas of the health sector and with health care providers-including radiologists-is an important aspect that needs to be addressed. Here, machine learning-supported image analysis tools could alert and direct physicians on findings that would then make it into a final report. The LSN analysis could be implemented as a simple test leading to the flagging of patients that qualify for additional workup. The data presented by Dioguardi-Burgio et al [1] expands and highlights the important role that MR scans can have in the future to detect a largely asymptomatic disease with a very high prevalence.

Funding Open Access funding enabled and organized by Projekt DEAL. JMS is partly funded by the European Union Innovative Medicines Initiative 2 (IMI2) Joint Undertaking under grant agreement 777377: LITMUS (Liver Investigation: Testing Biomarker Utility in Steatohepatitis).

\section{Declarations}

Guarantor The scientific guarantor of the editorial comment is Jörn M. Schattenberg, MD.
Conflict of interest JMS reports Consultancy: Boehringer Ingelheim, BMS, Genfit, Gilead Sciences, Intercept Pharmaceuticals, Madrigal, Merck, Novartis, Novo Nordisk, Nordic Bioscience, Pfizer, Roche, Sanofi, Siemens Healthcare GmbH.Research Funding: Gilead Sciences, Boehringer Ingelheim, Siemens Healthcare GmbH. Speakers Bureau: Falk Foundation. TE reports no conflict of interest.

Statistics and biometry No complex statistical methods were necessary for this paper.

Informed consent Written informed consent was not required for this study because this is an editorial comment.

Ethical approval Institutional review board approval was not required because this is an editorial comment.

Methodology

•Editorial Comment

Open Access This article is licensed under a Creative Commons Attribution 4.0 International License, which permits use, sharing, adaptation, distribution and reproduction in any medium or format, as long as you give appropriate credit to the original author(s) and the source, provide a link to the Creative Commons licence, and indicate if changes were made. The images or other third party material in this article are included in the article's Creative Commons licence, unless indicated otherwise in a credit line to the material. If material is not included in the article's Creative Commons licence and your intended use is not permitted by statutory regulation or exceeds the permitted use, you will need to obtain permission directly from the copyright holder. To view a copy of this licence, visit http://creativecommons.org/licenses/by/4.0/.

\section{References}

1. Dioguardi Burgio M, Sartoris R, Beaufrere A et al (2021) Liver surface nodularity on non-contrast MRI identifies advanced fibrosis in patients with NAFLD. Eur Radiol. https://doi.org/10.1007/ s00330-021-08261-6

2. Schattenberg JM, Lazarus JV, Newsome PN et al (2021) Disease burden and economic impact of diagnosed non-alcoholic steatohepatitis in five European countries in 2018: a cost-of-illness analysis. Liver Int 41(6): 1227-1242

3. Taylor RS, Taylor RJ, Bayliss S et al (2020) Association between fibrosis stage and outcomes of patients with nonalcoholic fatty liver disease: a systematic review and meta-analysis. Gastroenterology 158(6):1611-1625 e1612

4. Poynard T, Halfon P, Castera L et al (2007) Variability of the area under the receiver operating characteristic curves in the diagnostic evaluation of liver fibrosis markers: impact of biopsy length and fragmentation. Aliment Pharmacol Ther 25(6):733-739

5. Schattenberg JM, Straub BK (2020) On the value and limitations of liver histology in assessing non-alcoholic steatohepatitis. J Hepatol 73(6):1592-1593

6. Poynard T, Halfon P, Castera L et al (2007) Standardization of ROC curve areas for diagnostic evaluation of liver fibrosis markers based on prevalences of fibrosis stages. Clin Chem 53(9):1615-1622

7. Poynard T, Morra R, Ingiliz P et al (2008) Biomarkers of liver fibrosis. Adv Clin Chem 46:131-160 
8. Pickhardt PJ, Malecki K, Kloke J, Lubner MG (2016) Accuracy of liver surface nodularity quantification on MDCT as a noninvasive biomarker for staging hepatic fibrosis. AJR Am J Roentgenol 207(6):1194-1199

9. Lubner MG, Jones D, Said A, Kloke J, Lee S, Pickhardt PJ (2018) Accuracy of liver surface nodularity quantification on MDCT for staging hepatic fibrosis in patients with hepatitis $\mathrm{C}$ virus. Abdom Radiol (NY) 43(11):2980-2986

10. Sartoris R, Rautou PE, Elkrief L et al (2018) Quantification of liver surface nodularity at CT: utility for detection of portal hypertension. Radiology 289(3):698-707
11. Gidener T, Ahmed OT, Larson JJ et al (2021) Liver stiffness by magnetic resonance elastography predicts future cirrhosis, decompensation, and death in NAFLD. Clin Gastroenterol Hepatol 19(9):19151924.e6

12. Alqahtani SA, Schattenberg JM (2021) Nonalcoholic fatty liver disease: use of diagnostic biomarkers and modalities in clinical practice. Expert Rev Mol Diagn 21(10):1065-1078

Publisher's note Springer Nature remains neutral with regard to jurisdictional claims in published maps and institutional affiliations. 